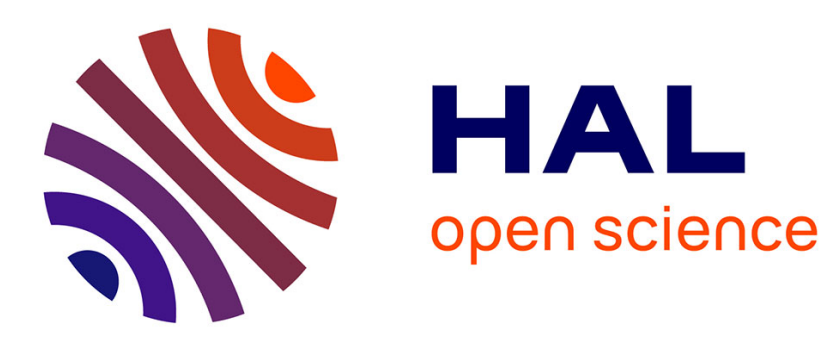

\title{
Analytic Phase Retrieval of Dynamic Optical Feedback Signals for Laser Vibrometry
}

\author{
Antonio Luna Arriaga, Francis Bony, Thierry Bosch
}

\section{To cite this version:}

Antonio Luna Arriaga, Francis Bony, Thierry Bosch. Analytic Phase Retrieval of Dynamic Optical Feedback Signals for Laser Vibrometry. IEEE Sensors Conference, Nov 2014, Valencia, Spain. hal01090363

\section{HAL Id: hal-01090363 https://hal.science/hal-01090363}

Submitted on 3 Dec 2014

HAL is a multi-disciplinary open access archive for the deposit and dissemination of scientific research documents, whether they are published or not. The documents may come from teaching and research institutions in France or abroad, or from public or private research centers.
L'archive ouverte pluridisciplinaire HAL, est destinée au dépôt et à la diffusion de documents scientifiques de niveau recherche, publiés ou non, émanant des établissements d'enseignement et de recherche français ou étrangers, des laboratoires publics ou privés. 


\title{
Analytic Phase Retrieval of Dynamic Optical Feedback Signals for Laser Vibrometry
}

\author{
Antonio Luna Arriaga ${ }^{1,2}$, Francis Bony ${ }^{1,2}$ and Thierry Bosch ${ }^{1,2}$ \\ ${ }^{1}$ CNRS, LAAS, 7 avenue du colonel Roche, F-31400 Toulouse, France \\ ${ }^{2}$ Univ de Toulouse, INP, LAAS, F-31400 Toulouse, France \\ alunaarr@laas.fr
}

\begin{abstract}
This paper presents a robust phase estimation to detect fringes of self-mixing signals for all feedback levels. It aims to avoid the need of restricting a particular working feedback regime for a sensor which is usually done by external optical/mechanical components. By removing the local offset from a set of fringes, their orthogonal representation can be obtained through the Hilbert transform allowing a unique calculation disregarding their shape in time. From it, it is possible to retrieve the displacement of a pointed target from a wide variety of usage conditions. The pertinence of the proposed approach for sensor implementation is also demonstrated.
\end{abstract}

\section{INTRODUCTION}

The development of non-contact sensors based on optical feedback interferometry or self-mixing (SM), continues to flourish mainly due to the compactness, low-cost and selfaligned characteristics of this technology in which a laser acts as a source and micro-interferometer at the same time [1].

In the context of target's displacement reconstruction, when a pointed surface moves away or towards a laser diode (LD), a small fraction of the backscattered light re-enters the active cavity of the laser interfering with the standing beam. The resulting fringe pattern related to the amount and frequency of the movement is obtained by monitoring the optical power variations either by a built-in photodiode, or directly from the junction voltage of the LD package. The equation describing such power variations is:

$$
P(t)=P_{0}\left\{1+m \cos \left[x_{f}(t)\right]\right\}
$$

where $P_{0}$ is the emitted optical power for the solitary LD, $m$ is a modulation coefficient, and $x_{f}$ the interferometric phase of the signal subject to back-reflections. SM fringes are not originated on free space, therefore a particular shape is characteristic of this phenomenon which is linked to the LD's linewidth enhancement factor $\alpha$ and mainly to a feedback coupling factor $C$. This adimensional parameter $C$ depends notably on the time of flight through the external cavity created between the LD and the target, as well as the effective power reflectivity on the surface. In practical applications, SM signals with coupling values between $0.1<C<1$ are said to be in weak feedback regime. They start with a quasi-sinusoidal shape (close to a usual interferometric waveform) from the lowest boundary and continue taking a sawtooth-like shape. Signals with $C>1$ are referred to be in moderate feedback regime. The appearance of hysteresis phenomenon affecting the zero-crossing of a set of fringes can be observed for increased values of $C$.
In order to retrieve the displacement of a remote target, a great variety of solutions can be found in literature to exploit this kind of interferometric signals. A tradeoff can be observed between adding external optical/mechanical components to the base SM configuration so as to perform simple signal processing over a restricted range of feedback coupling levels, or to perform more elaborated algorithms when the fully integrated nature of the SM sensing scheme wants to be privileged. Within the different signal processing techniques, usually it can be found algorithms performing for a particular feedback level. To increase the range of usage conditions, [2] implemented two different calculations in a sensor which selects the convenient procedure based on a custom peak detection criterion. Yet this selection requires an externally triggered mechanical auto-focus of about 2 seconds which penalizes its usage in dynamically changing feedback conditions.

Instead of using external triggering procedures when optical conditions change, it is desirable to have a unique algorithm suitable to handle different feedback levels. The approach proposed by [3] uses an adaptive threshold criteria to discriminate different feedback regimes. It then complements the previous iterative procedure by filtering procedures convenient to the identified regime. Recently, [4] signaled that the adaptive threshold approach needs to be improved when noisy SM signals are fed to its input. To this end, they proposed to remove a first prefilter block and instead perform a predefined piece-wise fringe detection. These two solutions require to acquire at least one period of the target oscillation to work properly, implying a huge quantity of system resources and thus also compromising their implementation for in-situ usage.

To illustrate this point we have tested the algorithm from [3] over a simulated SM signal corresponding to an amplitude displacement of $6 \lambda_{0}$ and a feedback coupling factor of $C=3$, being $\lambda_{0}$ the LD's wavelength emission. First, one period of a sawtooth-like signal [Fig.1(a)] (generated by the behavioral model described in [5]), was properly identified after 5 iterations of the adaptive threshold algorithm. Phase transitions of each fringe were processed in the correct direction of the target movement. However, in a second test with only half of the period of the same SM signal [Fig.1(b)], it can be observed that the adaptive threshold algorithm produced false transitions because this signal segment was processed with the filter convenient to weak feedback due to a wrong convergence of the iterative threshold. Notice that this use case may happen for example if a remote target decreases its frequency movent relative to a previous dimensioned value. 


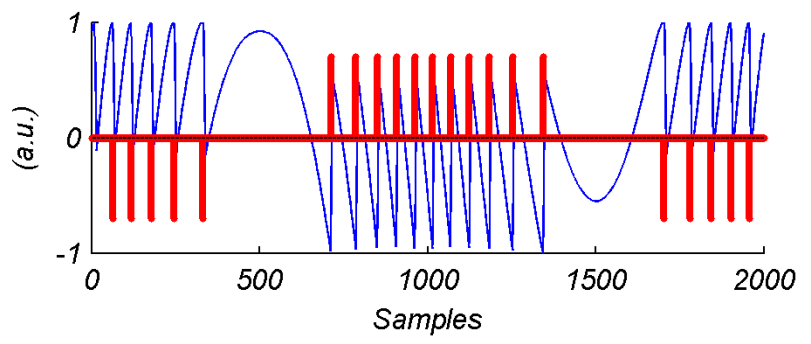

(a) $\mathrm{SM}$ with $C=3$ and one period of target's displacement.

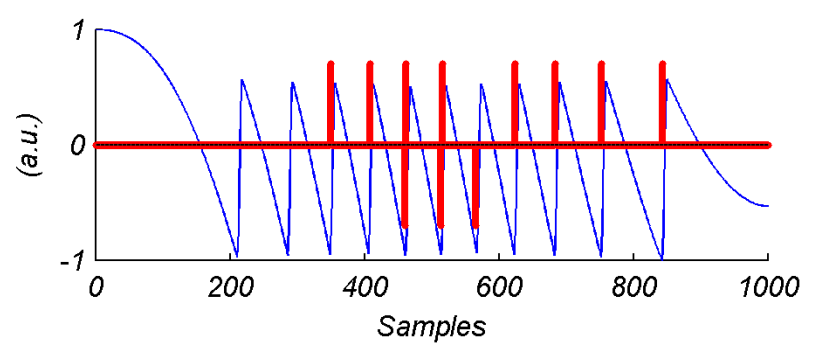

(b) The case when less than one period is processed.

Fig. 1: The adaptive threshold algorithm from [3] over two segments of a same SM signal.

Since an amplitude displacement of $\lambda_{0} / 2$ leads to the appearance of one fringe, an accurate measurement requires to detect all the acquired fringes. In this work we propose a unique calculation of phase transitions, allowing to detect SM fringes disregarding their shape in time and without the need of acquiring a full period of the target oscillation under measurement. In this way, a greater flexibility can be expected on the implementation of a displacement reconstruction sensor by SM.

\section{INSTANTANEOUS FREQUENCY OF SM SIGNALS}

Let us briefly outline the motivations and theory behind our proposed phase calculation by referring to Fig.2(a) where a simulated SM signal of $C=0.2$ from a sinusoidal displacement of amplitude $10 \lambda_{0}$ and $1 \mathrm{~Hz}$ of frequency, depicts the difficulty to exploit SM signals in time domain relying only on the fringes' shape. Performing a well dimensioned short time Fourier transform [Fig.2(b)], it can be observed the varying frequency of each of the fringes according to the target's movement. Furthermore, their associated even and odd harmonics clearly denote the non-sinusoidal characteristic shape of SM interferograms. Considering the case of a sharp sawtooth-like SM signal, the amount of harmonics associated to each of the fringes shall increase, spreading the signal's bandwidth. This example reveals the need to account such spectral variations along the time.

The notion of instantaneous frequency (IF) makes sense in this context as presented in [6]. In general terms, IF is understood as the rate of change of phase angle at a given time:

$$
I F=\frac{1}{2 \pi} \frac{d \phi(t)}{d t}
$$

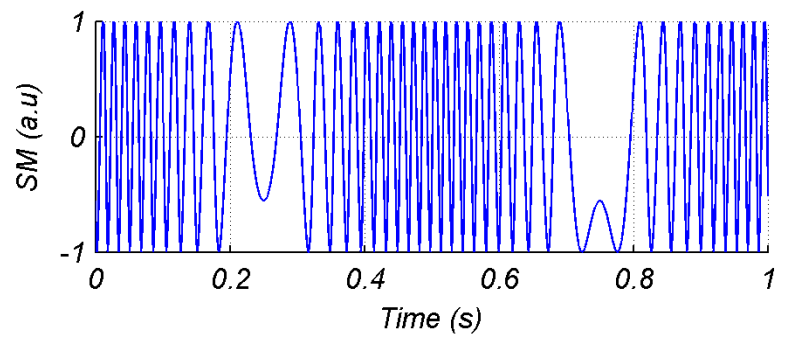

(a) SM with $C=0.2$ and one period of target's displacement.

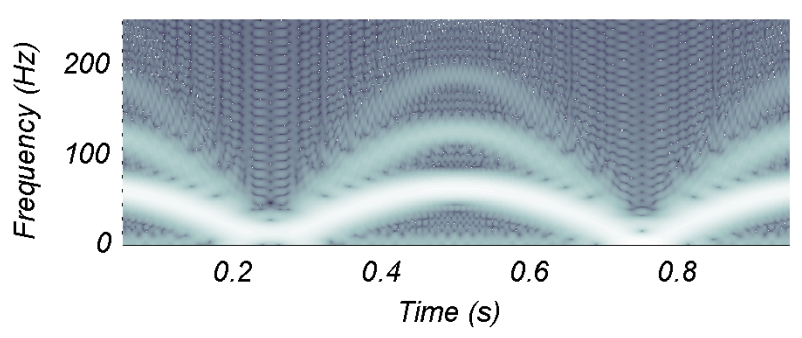

(b) Spectrogram for a sinusoidal movement and weak $C$.

Fig. 2: The need to account fringes' spectral variations along the time, specially in harmonic movements.

With the introduction of the concept of analytic signals from Gabor (see [7] for historical background), a conventional approach is to calculate the Hilbert transform $\mathcal{H}$, in order to generate a unique analytic representation in the complex plane of the data. On its simplest concept, $\mathcal{H}$ can be represented as a filtering process between the kernel $(-\pi t)^{-1}$ and the signal $s(t)$ of interest, i.e. a $90^{\circ}$ phase shift of the original signal over the orthogonal plane:

$$
\mathcal{H}[s(t)]=\frac{-1}{\pi t} * s(t)
$$

Expressed in frequency domain via Fourier transform $\mathcal{F}$ :

$$
\mathcal{F}\{\mathcal{H}[s(t)]\}=j \cdot \operatorname{sgn}(f) \mathcal{F}\{s(t)\}
$$

where $\operatorname{sgn}(f)$, the signum function is defined as 1 for positive frequencies, -1 for negative frequencies and null at zero.

Creating the analytic form of a SM signal can be seen as a means to provide further information aimed to simplify its exploitation. The formulation to obtain a SM signal in analytic form $P_{a}$ can be stated as:

$$
P_{a}(t)=P(t)+\operatorname{Im}\{\mathcal{H}[P(t)]\}
$$

From this representation, it is possible to exploit its orthogonality to calculate the phase from Eq. (2) by:

$$
\phi(t)=\arctan \left\{\frac{\operatorname{Im}\left[P_{a}(t)\right]}{\operatorname{Re}\left[P_{a}(t)\right]}\right\}
$$

In the following section we analyze this calculation over SM signals simulated at different feedback coupling levels. 


\section{ANALYTIC PHASE OF SM SIGNALS}

A conventional phase estimation (e.g. [3], [4]), consists in normalizing Eq. (1) in order to use:

$$
\hat{x}_{f}(t)=\arccos \left[P_{N}(t)\right]
$$

The resulting signal is high-pass filtered and thresholded to observe the transitions from one fringe to another. While this makes more sense for quasi-sinusoidal signals, it produces just a phase-mapped mirrored signal in the case of sawtooth-like signals from moderate feedback.

Since the detection of one fringe contributes to a phase unwrap of $2 \pi$, we extended the range of the estimated phase from Eq. (6) via a four-quadrant arctan function ( $\arctan 2)$, which is a variant of the trigonometric function arctan allowing to distinguish diametrically opposite directions from the input vectors for unambiguous results. In Fig.3 we compare these two calculations over the SM signal in weak feedback regime from Fig.2. In the case of conventional arccos phase estimation, SM maxima points [-1 1] agree [ $\pi$ 0] respectively, while our approach provides a linear fringe-phase representation over $(-\pi, \pi]$. It is also noticed that in this calculation, the values do not need to be normalized, so the usage of an automatic gain control circuit can be avoided.

In Fig.4 we have increased the feedback coupling value of the previous signal to obtain a moderate regime $(C=3)$ and a marked hysteresis phenomenon $(C=7)$. In both cases it can be observed that our calculation via $\arctan 2$ is not constant in amplitude, but instead presents a trending offset and a wraparound of instantaneous phase values. However, it can be realized that by calculating the absolute value of this instantaneous phase, waveforms similar to the $\arccos$ can be

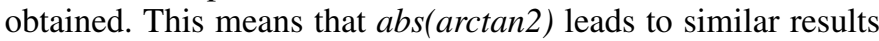
for transition detection like the conventional phase estimation.

We found an approach allowing to extend the usefulness of the proposed calculation, it consists in removing hysteresis phenomenon from the affected SM fringes in order to keep

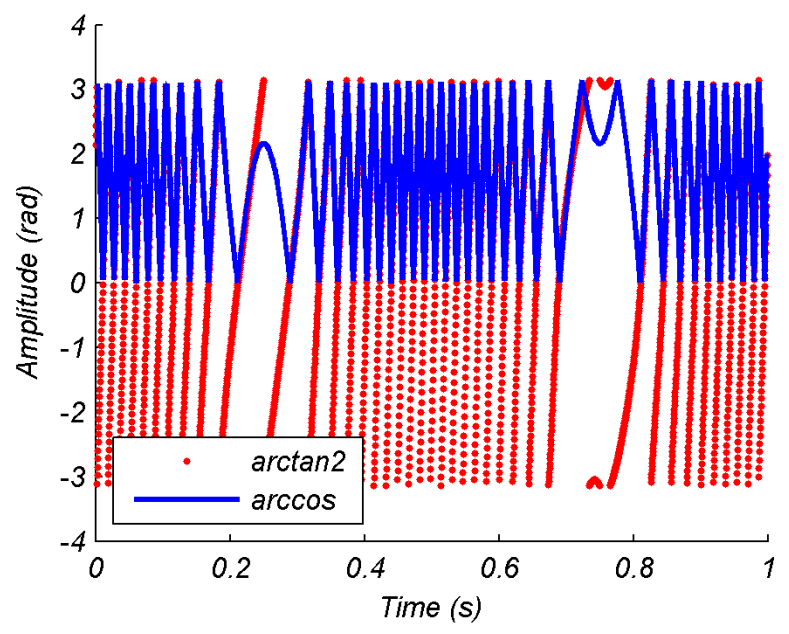

Fig. 3: Phase calculation comparison for the SM signal in weak regime $(C=0.2)$ from Fig.2(a).

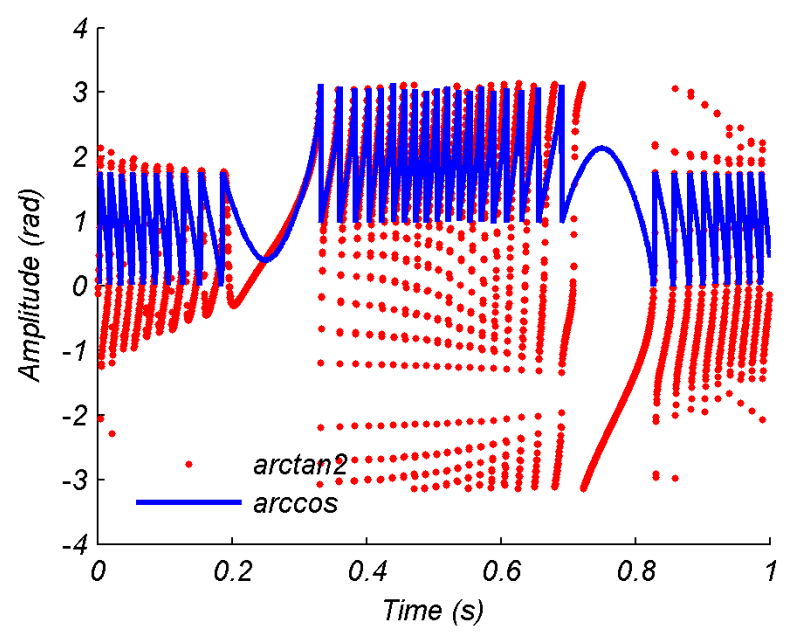

(a) SM signal with $C=3$ for sinusoidal target's displacement of $10 \lambda_{0}$ at $1 \mathrm{~Hz}$.

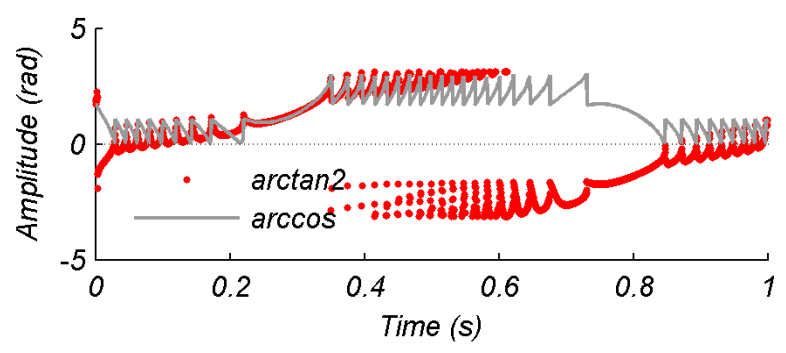

(b) SM signal with $C=7$ for sinusoidal target's displacement of $10 \lambda_{0}$ at $1 \mathrm{~Hz}$.

Fig. 4: Phase calculation comparison for a SM signal in moderate regime.

them centered to the zero-axis. A common calculation to reject this bias offset is to estimate a bandpass filter preventing low signal fluctuations as well as high frequency noise components. For convenience, we used a running average filter over a set of fringes of each target direction. Then, this averaged signal was subtracted to the hysteresis affected fringes like represented in Fig.5(a) for a simulated signal of $C=3$.

Referring to the regular analytic phase calculation of the signal in moderate feedback from Fig.4(a). It can be observed in Fig.5(b) that its equivalent centered signal produces an homogeneous phase estimation in an extended range as was observed for the case of weak feedback (Fig.3). According to Eq. (2), the phase derivative leads to the instantaneous frequency of the SM signal as denoted by the peaks of $2 \pi$ amplitude obtained for the centered SM signal. The presence of small amplitude peaks can be considered as a harmonics indication since they become more important in the case of moderate regime. By settling a fixed threshold to select these major peaks, increased robustness for fringe detection can be achieved disregarding their shape in time.

Once the fringes are segmented, the remaining point consists in assigning their direction to retrieve a target's movement. As a matter of fact, in SM literature there can be found several useful methods to reveal the direction of a 


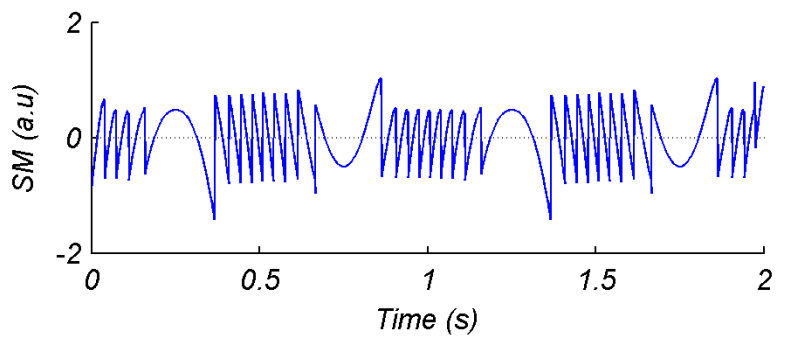

(a) $\mathrm{SM}$ with $C=3$ and subtracted hysteresis.

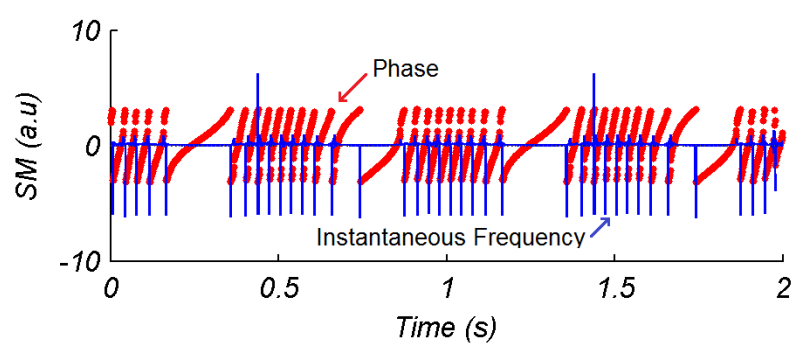

(b) Instantaneous frequency for zero-centered SM signal.

Fig. 5: Useful exploitation of SM signals in moderate regime through its analytic phase.

moving target. In our implementation, a simple procedure was conceived to save on calculation resources. First, the acquired signal is differentiated and converted into positive and negative impulsions according to their sign value. An example of this procedure is shown in Fig.6 where a SM signal in moderate regime $(C=3)$ produced a train of pulsed values. These values were then multiplied by the instantaneous frequency peaks denoted in Fig.5(b) to obtain thus the assigned direction for each phase transition. It must be said that this procedure needs to be improved in the case of noisy signals, however for a generic implementation it keeps a good agreement between the results and the complexity of calculations. We have tested this procedure for a variety of feedback conditions and observed a correct direction assignation by this simple procedure. The major improvement is on the robust detection of fringes as demonstrated hereafter.

\section{COMPARATIVE RESULTS}

We have implemented our proposed algorithm over the segment of the SM signal [Fig.1(b)] used to denote a drawback of the adaptive threshold algorithm from [3] (this is also expected for the algorithm in [4]). In Fig.7 it can observed that our calculation, denoted HT due to the Hilbert transform basis, is able to properly find the fringes on a small segment of acquired signal, while the previously analyzed algorithm produced wrong detections in the middle of the segment.

\section{Conclusion}

We have demonstrated that the usage of analytic SM signals allows a similar phase observation than the classical approach based on the inverse calculation of the cosine function, with the added value of avoiding the need for an automatic gain control for normalizing the input signals. We have also identified

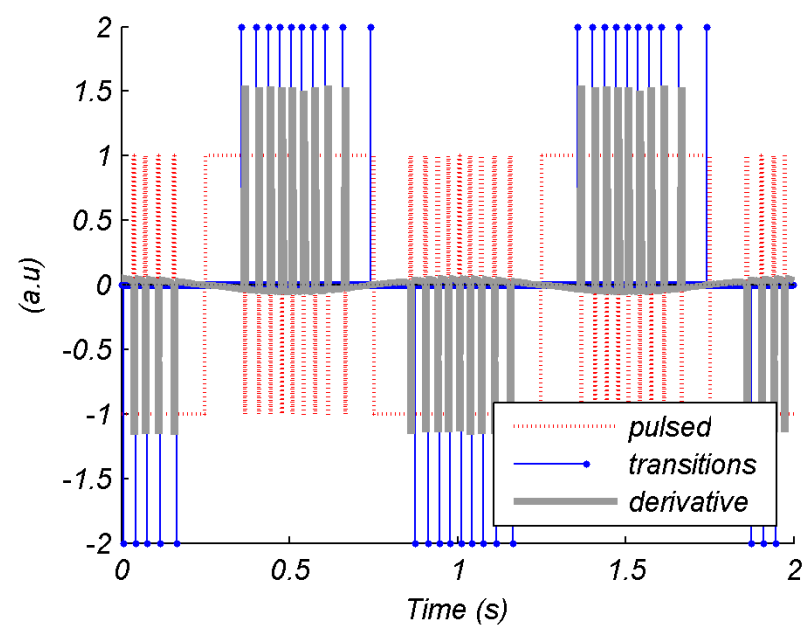

Fig. 6: Direction assignation of detected fringes for a SM signal in moderate feedback $(C=3)$.

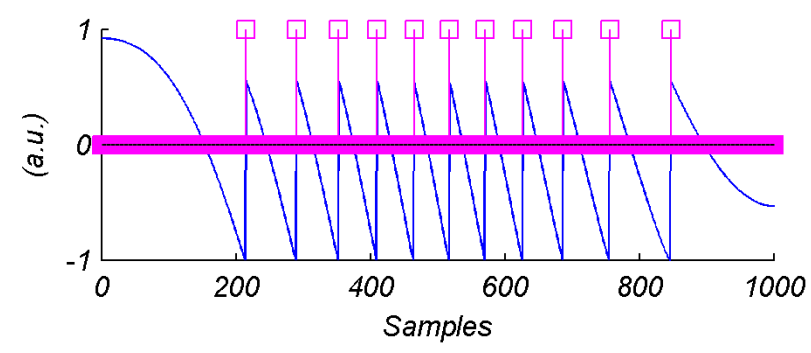

Fig. 7: The case when less than one period is processed by the algorithm from [3], is corrected by our proposal.

a method to detect the fringes from SM signals in either weak or moderate feedback avoiding the complex need of classifying. Our approach has been demonstrated to work for small segments of signals, and since it is based on filtering procedures, it is convenient to perform real-time displacement reconstruction.

\section{REFERENCES}

[1] S. Donati, "Developing self-mixing interferometry for instrumentation and measurements," Laser Photonics, vol. 6, no. 3, pp. 393 - 417, 2012.

[2] A. Magnani, A. Pesatori, and M. Norgia, "Self-mixing vibrometer with real-time digital signal elaboration," Applied Optics, vol. 51, no. 21, pp. 5318 - 5325, 2012

[3] U. Zabit, T. Bosch, and F. Bony, "Adaptive transition detection algorithm for a self-mixing displacement sensor," IEEE Sensors Journal, vol. 9, no. 12 , pp. 1879 - 1886, 2009.

[4] Z. Huang, C. Li, and X. Sun, "Piece-wise transition detection algorithm for a self-mixing displacement sensor," Chinese Optics Letters, vol. 11, p. 091203, 2013.

[5] G. Plantier, C. Bes, and T. Bosch, "Behavioral model of a self-mixing laser diode sensor," IEEE J. Quantum Electron., vol. 41, no. 9, pp. 11571167, sep. 2005.

[6] B. Boashash, "Estimating and interpreting the instantaneous frequency of a signal - part 1: Fundamentals," Proceedings - IEEE, vol. 80, pp. 520-538, 1992.

[7] S. L. Hahn, "The history of applications of analytic signals in electrical and radio engineering," in Proc. IEEE EUROCON, 2007. 